quollen, überhaupt unverändert geblieben, ja selbst der durch Kalilauge eine gelbe Farbe annehmende Gerbstoff ist noch in den Zellen vorhanden; erst beim Kochen derselben mit Wasser quillt die Intercellularsubstanz auf und gleichzeitig kann man beobachten, dass die bis dahin unverletzt gebliebene Hautschicht des Plasmas sich zusammenzieht und die Reste der Eiweisskörper in sich einschliesst.

Die Vortheile der osmotischen Maceration bestehen mithin darin, dass die Herausführung der Zuckerlösung bei einer Temperatur erfolgt, bei welcher die Intercellularsubstanz noch nicht quillt, welcher Umstand eine doppelte Bedeutung gewinnt: der Austritt der Zuckerlösung wird nicht erschwert und die Bildung löslicher, die Rübensäfte verunreinigender Pectinkörper wird unmöglich gemacht. (Sitzungsber. der k. Akad.d. Wiss. zu Wien. Math.naturu. Classe. L. Bd. 3. u. 4. Heft. UI. Abth. S. 442-453.) H. Ludvig.

\title{
Ein Mannaregen bei Karput in Kleinasien im März 1864.
}

Ueber denselben berichtet W. Haidinger, welcher vom $\mathrm{k} . \mathrm{k}$. Internuntius in Constantinopel, Freiherm von Prokesch.Osten, eine Partie von etwa 1 Pfund Wiener Gewicht dieser Manna zugeschickt erhielt, die in der Gegend nordwestlich von Diarbekir in Kleinasien im März 1864 gefallen war.

Die Türken nennen diese Manna Kudret bogdhasi, das Wundergetreide, und erzählen mehre solcher Fälle. Dic Leute mahlen es wie anderes Getreide und finden es nährend und wohlschmeckend.

Aus einern Berichte des Statthalters von Karput an den Gross-Vezir vom 12. Zilhidsche 1280 (17. Mai 1864) verdient über die näheren Umstände dieses Vorfalles das Folgende notirt zu werden: Dieses Getreide ist vor anderthalb Monaten während eines Regengusses bei dem Orte Schehid Duzi, östlich von Harput (Charput, Karput) zur Erde gefallen und einige Leüte haben von 3 bis 5 Oka davon eingesammelt. Später ist derlei Frucht auch in der Nähe von Malatia niedergegangen. Die Menge dieses Getreides ist wohl an sich unbedeutend, aber man hat von Alters her die Beobachtung gemacht, dass in Jahren der Dürre, wie wir deren hier hatten, auf einen Fruchtsegen in dem kommenden Jahre schliessen 
lasse, wenn ein derartiger Fruchtregen niedergeht. Die Einwohner sind daher in dankbarer Stimmung, die Getreidewucherer andererseits, durch frühere Erfahrungen gewitzigt, kommen mit ibren Getreidevorräthen hervor und so hat dieser Vorfall die Getreidepreise bedeutend sinken gemacht (den Kilo Weizen von 120 Piaster auf 80 herunter). Wir haben auch, Gott sei Dank, bereits wiederholten ausgiebigen Regen gehabt und an den meisten Saaten lassen sich die Wirkungen des Getreidesegens beobachten. Geruhen Eure Hoheit etc."

In dem 15. Bande II. Abtheilung der Verbandlungen (Nova Acta) der k. Leop. Carol. Akademie der Natur. forscher, Breslau und Bonn 1831, S. 351, findet sich über die Flechte, welche die Körner der Himmelsmanna bildet, eine Abhandlung Eduardi Eversmanni, D. Professoris Casaniensis, A. C. N. C. S., in Lichenem esculentum Pallasii et species consimiles adversaria. Cum tabula lapidi incisa et vivis coloribus picta (Acad. trad. die VIII. Mart. A. 1825); mit einem Nachtrage von Dr. Fr. L. Nees von Esenbeck. Haidinger selbst theilte ein ähnliches Ereigniss (einen Mannaregen) nach dem $\mathrm{Be}-$ richte von Siegfried Reissak in den "Berichten über die Mittheilungen von Freunden der Naturwissenschaften in Wien" mit.

Auch die vom Freiherrn von Prokesch-Osten übersandte Manna von Karput besteht aus rundlichen, mehr oder weniger Maulbeeren-, Erdbeeren- oder Himbeerentheilen ähnlichen Körnern einer Flechte, der Parmelia (Lecanora) esculenta Pallas, von blassgelblich-grauer Oberfäche, die grössten bis $3 / 4$ Zoll lang, 1/2 Zoll breit und dick (18 Millim. gegen 13 Millim.), doch die Mehrzahl kleiner. Sie schneiden sich mit einem scharfen Messer ganz leicht durch und erscheinen im Innern mehlig und vollkommen weiss, höchstens mit einem gelblichen Stiche. Die Körner stimmen im Allgemeinen gut mit der von Eversmann gegebenen Beschreibung, doch will Haidinger das ihm zur Verfügung stehende Material möglichst in der Art vertheilen, dass es weiterer wissenschaftlichen Forschungen dargebracht wird.

Eversmann gab sich vergeblich Mühe, Stücke zu finden, welche an andere Körper angewachsen gewesen wären. Die Manna von Karput zeigte in dieser Beziehung einige Abweichung. Zwar konnte $\mathrm{Haiding}$ er viele Körner derselben zerschneiden, ohne einen festen Kern als Unterlage anzutreffen. Aber bei einer vorläufigen Schau 
über zwei Drittel der erhaltenen Menge (ein Drittel hatte er bereits weggeschickt), traf er einige und zwanzig Stücke, nämlich der grösseren, die sich durch eine mehr eckige, kantige Form von den mehr rundlichen auszeichneten, welche einen wahren Stein im Innern hatten, einen solchen auch wohl nur unvollständig überkleideten, an einer Seite wohl gänzlich freiliessen. Einer derselben, welchen $\mathrm{Haidinger}$ entzwei schlug, bestand aus ganz frischem Granit, 1/4 Zoll (6 Millimeter) in jeder Richtung; die Rinde aus etwa 1 Millim. grossen und nur halb so dicken an einander schliessenden Körnchen bedeckt, die Quarz- und Orthoklas-Individuen sind ganz ansehnlich, 3-4 Millim. dick. Der Granit ist aber kein Geschiebe, sondern ein eckiges Bruchstück, nach allen Richtungen mit scharfen, nicht abgerundeten Kanten. Ein anderes Stück dieser Art hatte zur Masse feinkörnigen Kalkstein, heftig in Säuren brausend, ein anderes wieder feinkörnigen Quarz-Sandstein, andere mögen noch den verschiedensten Gebirgsarten angehören. So viel ist augenscheinlich, diese Mannaflechte stimmt mit andern Flechten, wie sie Steine überkleiden, ebenfalls überein. Dagegen ist diese Thatsache auf einer andern Seite wieder auffallender. Bei der leichten Flechte ist man gern bereit zuzugeben, dass sie durch einen Sturm, vielleicht erst durch einen cyclonenartigen Wirbel erhoben und sodann weit weggeführt werde; nebst den ganz leichten Stïcken muss aber nun bei dem Falle von Karput auch das schwerere vom Sturme mit fortgerissen worden sein. Mehre der übersendeten Steinchen wogen im Durchschnitte über 1/2 Grm. (1/30 Loth). Es ist dann natürlich, zu fragen, woher diese Massen der Flechte gekommen sind. Pallas, Eversmann fanden sie reichlich in den kirghisischen Steppen, namentlich den Montibus mugosaricis (Muchadschar der Karten) bis zum Embafluss. "Von dort bis Diarbekir ist eine Entfernung von etwa 280 geograph. Meilen. In dieser Voraussetzung müsste der Strich des Sturmes von N.O. gegen S.W. gerichtet gewesen sein. Fiel die Manna bei Karput mit Ostwind, so wäre eine mögliche ursprüngliche Fundstätte die grosse turkomanische Wüste im Süden des Khanats Khiwa, östlich vom südlichen Theile des Caspisees, und dann betrüge die Entfernung etwa gegen 240 geogr. Meilen. Ein "zerstörender Orkan" (devastating hurricane) ist nach Rouse's anemometrischer Tabelle auf 100 engl. Meilen die Stunde geschätzt, also in runder Summe etwa 20 
deutsche. Er müsste, nachdem er einmal die Mannaflechten erfasst, doch etwa 12 bis 14 Stunden in gleicher Strömung fortgedauert haben, was allerdings wohl inner-4 halb der Grenzen liegt, welche man für eine solche doch immer ungewöhnliche Erscheinung voraussetzen könnte. Die Angabe des Ortes der Fälle von 1824, 1828, 1841, 1846 und 1864 liegen nahe in einer westöstlichen Linie Wan, Karput, Malatia, Jenischehir. Persien wird nach Parrot von Reissek nur im Allgemeinen angeführt; Aderbidschan, Tabris läge allerdings auf derselben Linie.

Merkwürdig ist das von Nees von Esenbeck angeführte Ergebniss der chemischen Analyse der von Parrot mitgetheilten persischen Parmelia esculenta durch Friedemann Göbel (Schweigger's Journ. fur Chemie $u$. Phys. 1830. Bd.3. H. 4. S. 393). Nicht Stärkemehl ist der weisse Körper, wie man auf den ersten Blick vermuthen möchte, wenn man von Vermahlen und Brodbacken hört, sondern je 65,91 Proc. oxalsaurer Kalk und das Nährende würde vornehmlich auf den 23 Proc. Gallerte beruhen.

Die übrigen Bestandtheile waren nach Fr. Göbel: 1,73 Proc. in Aether lösliches, grünlich-gelbes, Chlorophyll enthaltendes Weichharz, von kratzendem Geschmack, 1,75 Proc. in Alkohol lösliches, geruch- und geschmackloses Weichharz, 1 Proc. einer in Alkohol und Wasser löslichen, bitterlich schmeckenden Substanz, 2,5 Proc. Inulin, 3,25 Proc. Flechtensäure (und 0,84 Proc. Verlust).

Höchst anziebend, gerade wegen dieses überraschend hohen Gehalts an oxalsaurem Kalk, kam $\mathrm{H}$ aidinger als ein im Augenblicke doppelt dankenswerthes Geschenk durch Prof. L. Radik ofer in München für Herrn Dr. Georg Holzner die Inaugural-Abhandlung des Letzteren $\mathrm{zu}$, in welcher derselbe in umfassender Weise die Krystalle in den Pflanzenzellen darlegt und den Nachweis liefert, dass sie sämmtlich oxalsaurer Kalk sind, aber theíls dem quadratischen (pyramidalen), theils dem klinorhombischen (augitischen) Systeme angehören. Sie werden namentlich in den Rindengebilden verfolgt. Herr Dr. Holzner erwähnt keiner Untersuchungen an Flechten; Haidinger wird ihm eine Probe der Parmelia des neuen Falles von Karput mittheilen.

Dr. Th. Kots chy theilt mit, dass er durch den General Jus $\mathbf{s}$ f vor zwei Jahren eine Plechte, ähnlich der asiatischen, erhielt, welche Chlorangium Jussuffii oder Parmelia esculenta $\beta$ Jussuffii genannt wird. 
In Peter von Tchihatcheffs Asie mineure 3. Botanique II. S. 662 finden sich folgende Angaben für Parmelia esculenta Endl.; von Fundstätten: "Agro Byzantino"; nach Rigler's: "die Türkei und ihre Bewohner", Lycaonia planitiebus excelsis aridisque nach Tchihatscheff's eigenen Beobachtungen und "Sahara Algeriens* nach E. Cosson in dem Bull. de la Soc. bot. IV. 473. Eine Angabe über die Häufigkeit des Vorkommens ist nicht beigefügt, doch würde allerdings aus den dürren Hocbebenen Karamaniens die Entfernung bis Malatia und Karput 60-80 Meilen, und zwar mit WSW-Winden, eine wenigstens nähere Quelle zu der Erscheinung bezeichnen, als der Bezug aus dem viel entfernteren Osten oder Nordost.

Ueber die algierische Manna theilte Hofrath L. Reichenbach von Dresden in einem Schreiben an H. A. Senoner einige Notizen mit. Er hatte Proben derselben von dem damals in Algier stationirten GeneralStabsarzt der französischen Armee in Afrika Dr. Guyon erhalten, welcher über die Orte des Vorkommens schon 1852 Nachricht gab. Die Beni M'zab hatten die Flechte mit Sand bereits 1835 nach Algier gebracht. Zehn Jahre später wurde sie durch eine unter dem Befehle des Herzogs von Aumale nach dem El Berda und dem Teli in der öden Gegend zwischen dem Djehel Dira und Djebel Amour marschirenden Armee-Colonne in grosser Menge angetroffen. In der Wildniss sind die Pferde, Kameele, Gazellen und andere Thiere lüstern nach dieser Flechte, auch der Mensch lässt sie sich gefallen; doch fielen die Versuche, wirkliches Brod daraus zu erzeugen, sogar mit Zusatz von Mehl, nicht hinlänglich günstig aus, um die grossen Hoffnungen zu erfüllen, welche mun erst auf sie gesetzt hatte. (Wien. Sitzungsber. der k. k. Akad. der Wiss. Math.-naturw. Cl. 50. Bd. 2.Heft. II. Abth. S. 170-177.)

H. Ludwig.

\section{Verbindungen des Mannits mit den alkalischen Erden.}

G. Hirzel hat die von Ubaldini bereits analysirten Verbindungen des Mannits mit den alkalischen Erden nochmals dargestellt und hat für dieselben andere Formeln berechnet. Nach seiner Untersuchung sind diese Verbindungen folgendermassen zusammengesetzt: 